\title{
A Modelica Library for Spacecraft Thermal Analysis
}

\author{
Tobias Posielek ${ }^{1}$ \\ ${ }^{1}$ Institute of System Dynamics and Control, DLR German Aerospace Center, Oberpfaffenhofen, Germany \\ tobias.posielek@dlr.de
}

\begin{abstract}
In spacecraft missions it is vital to maintain all spacecraft components within their required temperature limits. Thus, a model incorporating all main heat fluxes acting on the spacecraft is necessary to allow for the design of a thermal control subsystem. This paper introduces the thermal space systems library which implements common models of radiation and thermal components of a spacecraft. Special effort is put into the calculations of the angles describing the orientation of the spacecraft with respect to sun and earth. Issues occurring due to the recalculation of the angles in each time step are shown and methods for their determinations are given.
\end{abstract}

Keywords: space modeling, thermal modeling, angle determination

\section{Introduction}

In spacecraft engineering, it is essential to ensure that all components operate in their appropriate temperature range to avoid malfunction and equipment breakage. Therefore, an analysis of the thermal dynamics is a necessity to design the required thermal control (Gilmore and Bello 1994), (Meseguer, PérezGrande, and Sanz-Andrés 2012), (Fortescue, Swinerd, and Stark 2011). A rigorous description of the thermal system is difficult as it has to incorporate the orbit and the orientation of the spacecraft during the mission, as well as the sun's position and the dissipated energy within the spacecraft. The modelling of the thermal system is a present topic of interest (Ruan, $\mathrm{Hu}$, and Sun 2017) (Lefeng et al. 2017) (Qian et al. 2015). Approaches of various complexity exist to design the thermal control. Simple design approaches consider only static worst case scenarios to account for degradation and orbit thermal dynamics (Larson and Wertz 1991). Other methods use analytical models to obtain the dynamic evolution of the temperature over the course of multiple orbits (Tsai 2004).

The proposed library allows the simulation of the complete spacecraft system including the thermal system as well as the electric and mechanical system providing the dissipated energy and spacecraft orientation dependent on the spacecraft mission. The library is proposed in view of simple analytical mod- els. Generally, a spacecraft is modelled by a huge number of nodes with different heat fluxes acting on each. We will only model the most important nodes e.g. each surface may be modelled as a node for a cuboid spacecraft. For each of these nodes the temperature dynamic is determined by the dynamic of its adjacent nodes and the four main heat flows due to the environment. One main point which will be illuminated is the calculation of the angle between the spacecraft surfaces and its surroundings. As the attitude of spacecraft is usually not known a priori and determined online, suitable methods to calculate this angle are proposed. The library is created in view of earth orbiting spacecraft. However, the library can also be used for simulations of spacecraft leaving earth orbit as long as modifications regarding the coordinate systems and approximations, such as shadow calculations, are made. The proposed library uses the other Modelica-based libraries of the Institute of System Dynamics and Control at the DLR German Aerospace Center such as the Environment library (Briese, Klöckner, and M. Reiner 2017) and SpaceSystems library (M. J. Reiner and Bals 2014). The library is created as an in-house library as a part of the design of an energy management for spacecraft. Section 2 introduces the essential fundamentals for the thermal dynamics. Section 3 gives details to the Modelica implementation and in Section 4 an example scenario is simulated to show the functionality of this library.

\section{Fundamentals}

This section introduces the coordinate systems, heat fluxes, solar angles, form factor and shadow function necessary to simulate the spacecraft thermal system.

\subsection{Coordinate Systems}

The Earth-Centered Inertial (ECI) Frame is defined such that the $x^{\mathrm{I}}$-axis points in direction of vernal equinox, this is the intersection between the equator and the sun's apparent orbit during spring. The $z^{\mathrm{I}}$-axis is parallel to the mean Earth's rotation axis and towards the North Pole and the $y^{\mathrm{I}}$-axis completes right handed coordinate system. For all following reference frames the rotation matrix to ECI coordinates is given by their coordinate axes. Each coordinate system will be denoted with a superscript which will 


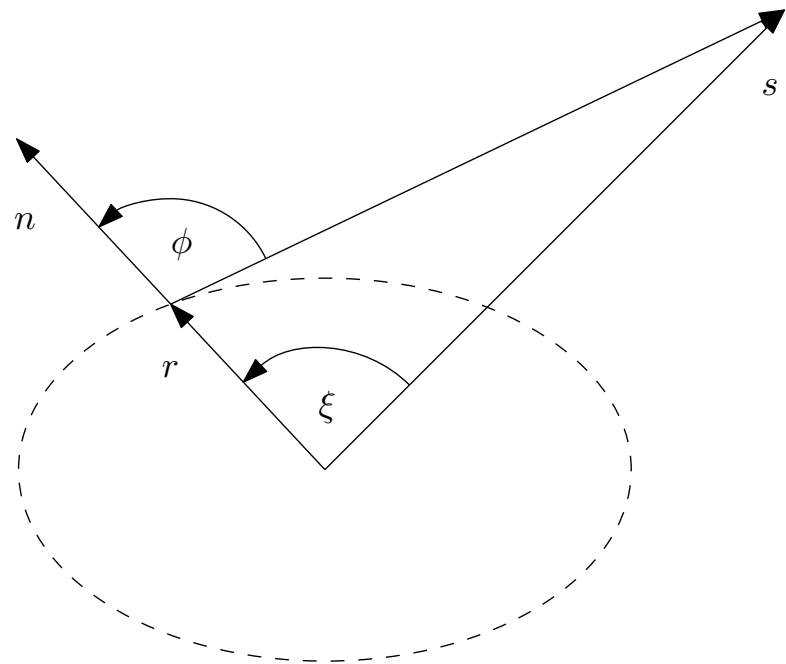

(a) Main angles influencing the temperature evolution in a spacecraft. The solar zenith angle $\xi$ is defined as the angle between the vector to the spacecraft $r$ and the vector pointing to the sun $s$. The normal solar angle $\phi$ describes the angle between the normal of a spacecraft surface $n$ and the vector pointing from spacecraft to the sun. The dotted line describes the spacecraft orbit.

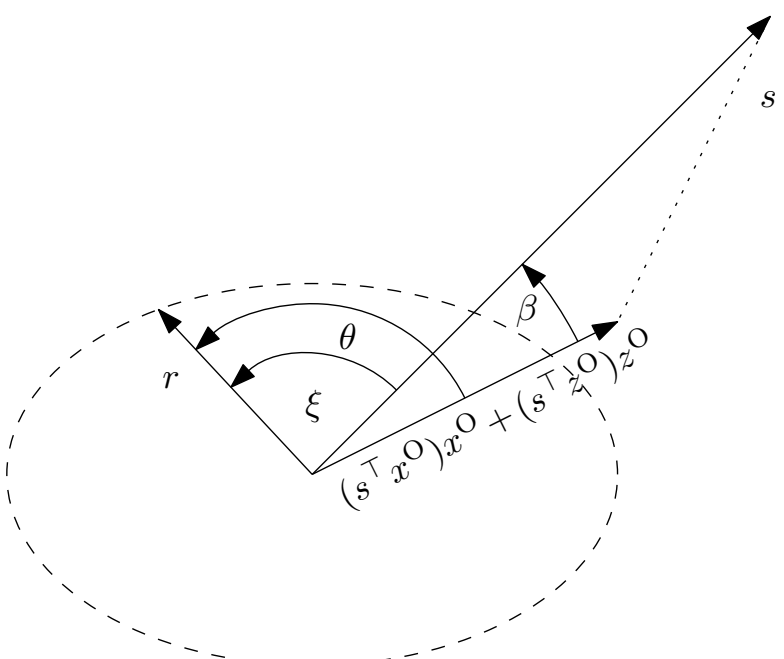

(b) Angles describing the influence of the solar zenith angle $\xi$. The solar noon angle $\theta$ describes the angle between the vector pointing to the spacecraft $r$ and the solar noon, i.e. the vector pointing to the sun projected on the orbit plane $\left(x^{\mathrm{O}} s\right) x^{\mathrm{O}}+\left(z^{\mathrm{O}} s\right) z^{\mathrm{O}}$. The beta angle $\beta$ is defined as the angle between the orbit plane and the vector pointing to the sun $s$.

Figure 1. Solar Angles

be used for the notation of their coordinate axes and rotation matrices. We denote $T^{\mathrm{S}, I}=\left[\begin{array}{lll}x^{\mathrm{S}} & y^{\mathrm{S}} & z^{\mathrm{S}}\end{array}\right]^{\top}$ as the transformation from ECI coordinates to an arbitrary coordinate system with superscript $S$. So for $r^{\mathrm{I}}$ in ECI coordinates the transformed vector $r^{\mathrm{S}}$ is calculated via

$$
r^{S}=T^{\mathrm{S}, I} r^{I} .
$$

The orbit frame is defined for a spacecraft in an elliptical orbit with position $r^{\mathrm{I}}(t)$ and velocity $v^{\mathrm{I}}(t)$ in inertial coordinates by the $y^{\mathrm{O}}$-axis which is normal to the orbit plane in direction of negative angular momentum, the $z^{\mathrm{O}}$-axis which points to geocentric nadir and the $x^{\mathrm{O}}$-axis which completes the right handed coordinate system and is for circular orbits in direction of velocity. We omit the time argument on the right hand side and obtain the transformation from ECI coordinates to orbit coordinates as

$$
T^{\mathrm{O}, \mathrm{I}}(t)=\left[\frac{r^{\mathrm{I}} \times\left(r^{\mathrm{I}} \times v^{\mathrm{I}}\right)}{\left\|r^{\mathrm{I}} \times\left(r^{\mathrm{I}} \times v^{\mathrm{I}}\right)\right\|} \quad-\frac{r^{\mathrm{I}} \times v^{\mathrm{I}}}{\left\|r^{\mathrm{I}} \times v^{\mathrm{I}}\right\|} \quad-\frac{r^{\mathrm{I}}(t)}{\left\|r^{\mathrm{I}}(t)\right\|}\right]^{\top} .
$$

\subsection{Environmental Heat Fluxes}

Mainly four environmental heat fluxes are acting on a spacecraft surface, namely the heat flux due to direct solar irradiation, the solar radiation reflected by the earth, the radiation of the earth emitted in the infra-red spectrum and the radiation of the spacecraft emitted to deep space (Larson and Wertz 1991),(Meseguer, Pérez-Grande, and SanzAndrés 2012). Each of these fluxes and its calculation is introduced in this section.

\subsubsection{Direct Solar}

The solar radiation is the main factor influencing temperature changes of the spacecraft. A solar constant $G_{s 0}$ is defined as in (Meseguer, Pérez-Grande, and Sanz-Andrés 2012) which gives the mean solar irradiance acting on a unit area perpendicular to the solar rays in a distance of 1 ua where ua denotes the astronomical unit. As the amount of irradiance crossing spherical surfaces with different radii is assumed to be constant, the solar irradiation $G_{s}$ scales with distance as

$$
G_{s}(d)=G_{s 0} \frac{d_{0}^{2}}{d}
$$

where $d$ is the distance in astronomical units and $d_{0}=1$ ua. The solar energy is mostly distributed in visual and short wavelength infra-red (Larson and Wertz 1991). This allows for surfaces which are very reflective in the solar spectrum but highly emissive to long wavelength infra-red. A simple analytical model incorporates the angle $\phi=\phi(n, r, s) \in[0, \pi]$ between the surface normal and the sun and the shadow of the earth described by the shadow coefficient $\nu=\nu(r, s) \in[0,1]$ introduced in Section 2.5. Then the acting solar flux reads

$$
Q^{\text {sun }}= \begin{cases}\alpha G_{s}\left(\frac{\|s-r\|}{1 \text { ua }}\right) A \cos (\phi) \nu & \text { if } 0<\phi<\frac{\pi}{2} \\ 0 & \text { if } \frac{\pi}{2}<\phi<\pi\end{cases}
$$

where $\alpha$ denotes the solar absorptance of the surface and $A$ the area of the surface. 


\subsubsection{Albedo}

By albedo we denote the part of the solar radiation which is reflected by the earth or scattered by the planet surface and atmosphere. Combining the simple models from (Larson and Wertz 1991) and (Meseguer, Pérez-Grande, and Sanz-Andrés 2012) we obtain

$$
Q^{\mathrm{alb}}= \begin{cases}\rho_{\mathrm{alb}} \alpha G_{s}(d) A F_{\text {form }} \cos (\xi) & \text { if } 0<\xi<\frac{\pi}{2} \\ 0 & \text { if } \frac{\pi}{2}<\xi<\pi\end{cases}
$$

where $\rho_{\text {albedo }} \in[0,1]$ is the albedo coefficient. This coefficient can vary over the course of an orbit and depends on the orbits inclination. This model incorporates the solar zenith angle $\xi(s, r) \in[0, \pi]$, the angle between the sun and the spacecraft, and a form factor $F_{\text {form }}(r, n)$ defined in Section 2.4 to describe the part of the radiation that actually strikes the spacecraft surface.

\subsubsection{Planetary Radiation}

As planetary radiation we denote the thermal radiation which is emitted by the planet as long wavelength infra-red radiation. The emitted radiation can be calculated as the absorbed solar radiation of the planet minus the radiation emitted via albedo. Then, by assuming the planet to be a black body we obtain the planetary infra-red thermal heat acting on a sufrace of a spacecraft as in (Meseguer, Pérez-Grande, and Sanz-Andrés 2012)

$$
Q^{\text {planet }}(r, n)=\varepsilon A F_{\text {form }}(r, n) \sigma T_{p}^{4}
$$

where $T_{p}$ denotes the black body temperature of the earth, $\sigma$ the Stefan-Boltzmann constant and $\varepsilon$ the infra-red emissivity of the surface. Instead of using the black body temperature of the earth (5), it is often written as

$$
Q^{\text {planet }}(r, n)=\varepsilon A F_{\text {form }}(r, n) I_{\mathrm{IR}}
$$

where $I_{\mathrm{IR}}$ is the intensity of earth infra-red flux to account for the variation of $Q^{\text {planet }}$. Note that $I_{\mathrm{IR}}$ is actually not a constant but also varying over the course of an orbit. However, the variation of $I_{\mathrm{IR}}$ is small in comparison to the albedo variation.

\subsubsection{Radiation to Deep Space}

The outer surfaces of a spacecraft are radiatively coupled to space. The energy of the reradiation to space is usually in the long wave infra-red spectrum and can be described by

$$
Q^{\mathrm{ds}}=\varepsilon A \sigma T^{4}
$$

where $T$ denotes the temperature of the surface.

These four heat fluxes are the main environmental heat fluxes acting on the spacecraft. Other fluxes dues to the environment exist but are neglected in the analysis due to their minor influence on most spacecraft. Numerical values for the parameters describing the solar absorptivity and infra-red emissivity of different surface can be found in the literature such as (Larson and Wertz 1991). Hot and cold case scenario parameters for $\rho_{\mathrm{alb}}, I_{\mathrm{IR}}$ and $G_{s}$ dependent on the orbit and can be found in (Larson and Wertz 1991). Formula to describe the solar angles $\phi, \zeta$, the form factor $F_{\text {form }}$ and the shadow coefficient $\nu$ are introduced in the following sections.

\subsection{Thermal Angles}

For the calculation of solar, albedo and infra-red irradiation, different angles describing the position of the sun and the attitude of the surface are of interest. These angles are visualized in Figure 1. Figure 1a shows the zenith angle $\xi$ and normal solar angle $\phi$ which are the angles influencing the generation of heat and Figure $1 \mathrm{~b}$ the solar noon angle $\theta$ and beta angle $\beta$ which can describe the influence of the solar zenith angle as will be explained later. In this section, we define these angles, show the relations between them and introduce two different ways to calculate these angles.

\section{Problem Formulation}

Usually two vectors $v_{1}$ and $v_{2}$ are given in a reference coordinate system. In order to calculate the angle between these two vectors, it may seem advantageous to use the scalar product as in formula (8) as you do not need any other rotations or coordinates system and use only the property of the scalar product. This however, gives only angles between $[0, \pi]$ which is sufficient for many calculations which use uneven functions but it leads to undesired results when rotations are considered as illustrated in Figure 3. In this figure the angle between the vectors $v_{1}$ and $v_{2}(t)$ is displayed on the left hand side over the course of a full uniform planar rotation illustrated in the middle. The angle moves between 0 and $\pi$ which makes no unique identification of the position of $v_{2}$ from the angle possible. Note that in the control context, the uniqueness issue can be solved by using quaternions which use the normal axes of the rotation as additional information. On the right hand side, the desired angle evolution is displayed which ensures the bijection between position and angle in a single rotation. In order to achieve this angle definition between $(-\pi, \pi]$, we use the properties of cylinder coordinates. Such a definition is simple if a coordinate system is constructed which $x-y$-plane describes the rotation plane or if the reference frame can be rotated on the rotation plane. Note however, that only a minimum of information about the rotation is known and one can only rely on the current value $v_{1}(t)$ and $v_{2}(t)$ but not on a closed description of the functions $v_{1}(\cdot)$ and $v_{2}(\cdot)$. This information has to be used to construct 


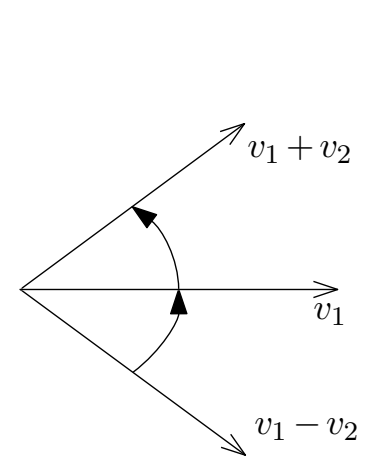

(a) Angle definition using (b)

(8)

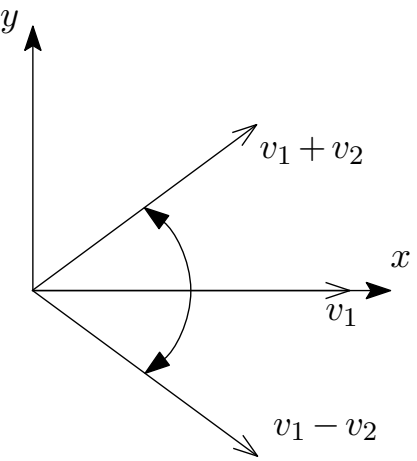
coordinate system (9)

Figure 2. Different angle definitions

the same coordinate system at every time step over the course of the rotation. Problems using intuitive coordinate systems definitions are illustrated in Figure 5 and 4 . Thus, an additional vector is necessary to construct this coordinate system. In the case of orbit rotations this vector comes by the cross product of velocity and position.

\section{Angle Definitions}

The most intuitive definition defines the angle $\theta \in$ $[0, \pi]$ as the smaller positive angle between the two vectors $v_{1}, v_{2} \in \mathbb{R}^{3}$ as

$$
\theta=\angle\left(v_{1}, v_{2}\right):=\cos ^{-1}\left(\frac{v_{1}^{\top} v_{2}}{\left\|v_{1}\right\|\left\|v_{2}\right\|}\right)
$$

where $\cos ^{-1}$ denotes the inverse of the cosine with a domain of $[0, \pi]$. This definition is sufficient for most purposes especially if only the cosine of an angle is of interest. However, as a result the angle between $v_{1}$ and $\alpha v_{1}+v_{2}$ is the same as between $v_{1}$ and $\alpha v_{1}-v_{2}$ with $v_{1}^{\top} v_{2}=0$ and $\alpha \in \mathbb{R}$ which is undesirable in view of planar rotations as illustrated in Figure 2a. This flaw can be overcome by using a cartesian coordinate system and its polar coordinate representation.

For cartesian coordinate system with axes $x, y$ and $z$ represented by its transformation matrix $T=$ $\left[\begin{array}{lll}x & y & z\end{array}\right]$ so that $T v_{1}$ and $T v_{2}$ are in the $x$-y-plane, we define the angle $\theta \in(-\pi, \pi]$ by

$$
\begin{aligned}
\theta=\angle^{x}\left(v_{1}, v_{2}\right):= & \operatorname{atan} 2\left(e_{2} T v_{2}, e_{1} T v_{2}\right) \\
& -\operatorname{atan} 2\left(e_{2} T v_{1}, e_{1} T v_{1}\right) .
\end{aligned}
$$

where $e_{i}$ denotes the $i$-th unit vector in $\mathbb{R}^{3}$ and atan 2 the extension of the atan function as

$$
\operatorname{atan} 2(b, a):=\left\{\begin{array}{ll}
\operatorname{atan}\left(\frac{b}{a}\right) & \text { if } a>0 \\
\operatorname{atan}\left(\frac{b}{a}\right)+\pi & \text { if } a<0 \wedge b \geq 0 \\
\operatorname{atan}\left(\frac{b}{a}\right)-\pi & \text { if } a<0 \wedge b<0 \\
\frac{\pi}{2} & \text { if } a=0 \wedge b>0 \\
-\frac{\pi}{2} & \text { if } a=0 \wedge b<0 \\
\text { undefined } & \text { if } a=0 \wedge b=0
\end{array} .\right.
$$

We use the superscript $x$ in $\angle^{x}$ to reference to the corresponding $x-y-z$-coordinate system which describes $T$. This definition gives for planar rotations angles the results as desired and is illustrated in Figure $2 \mathrm{~b}$. The angle between between $v_{1}$ and $v_{1}+v_{2}$ and $v_{1}$ and $v_{1}-v_{2}$ have different signs in comparison to Figure 2a.

With this definition we can describe the angles for planar rotations by using an at the beginning established coordinate system with the mentioned properties. However, as the desired reference coordinate systems for the calculations of the angles are subject to slow changes, it is necessary to redefine the coordinate system at every point of time. This means the coordinate axes have to be constantly recalculated. Clearly, it is desirable to obtain continuous axes that do not experience a change of sign. Furthermore, the coordinate system shall be right handed and use only information about the current point of time.

By the definition of the cross product, it is sufficient to use only two vectors $v_{1}$ and $v_{2}$ to define a coordinate system via

$$
\begin{aligned}
& x=\frac{v_{1}}{\left\|v_{1}\right\|}, \\
& y=z \times x, \\
& z=\frac{v_{1} \times v_{2}}{\left\|v_{1} \times v_{2}\right\|} .
\end{aligned}
$$

However, for a constant $v_{1}$ but a rotating $v_{2}$ the $y$ - and $z$-axis change their direction when $v_{1}$ and $v_{2}$ become parallel as can be seen in Figure 4.

Consider the Gram Schmidt process as a way to construct the coordinate system with

$$
\begin{aligned}
& x=\frac{v_{1}}{\left\|v_{1}\right\|}, \\
& y=\frac{v_{2}-\left(x^{\top} v_{2}\right) x}{\left\|v_{2}-\left(x^{\top} v_{2}\right) x\right\|}, \\
& z=\frac{v_{3}-\left(x^{\top} v_{3}\right) x-\left(y^{\top} v_{3}\right) y}{\left\|v_{3}-\left(x^{\top} v_{3}\right) x-\left(y^{\top} v_{3}\right) y\right\|} .
\end{aligned}
$$

However, with this definition it cannot be guaranteed that the resulting coordinate system is right handed as illustrated in Figure 5.

We combine these two methods in order to obtain a continuous right handed coordinate system. 


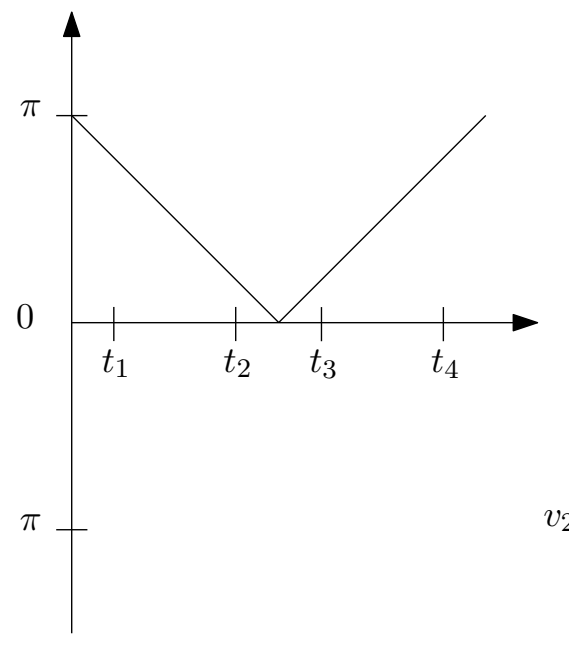

Scalar product angle definition

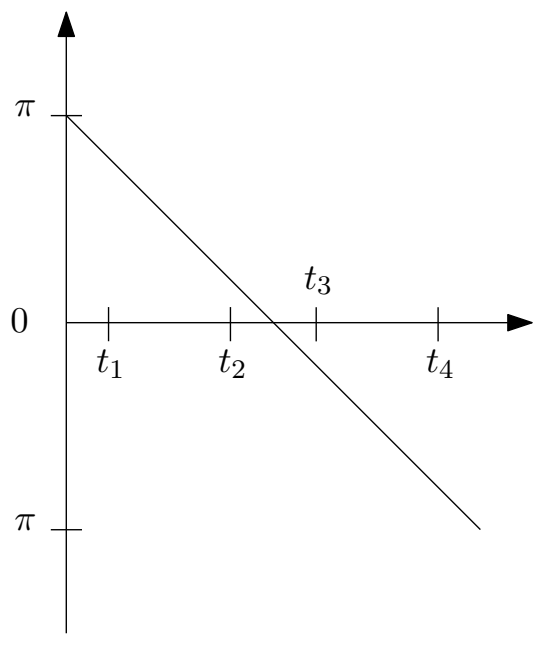

Planar angle definition

Figure 3. Angle of a planar rotation described by two different definitions
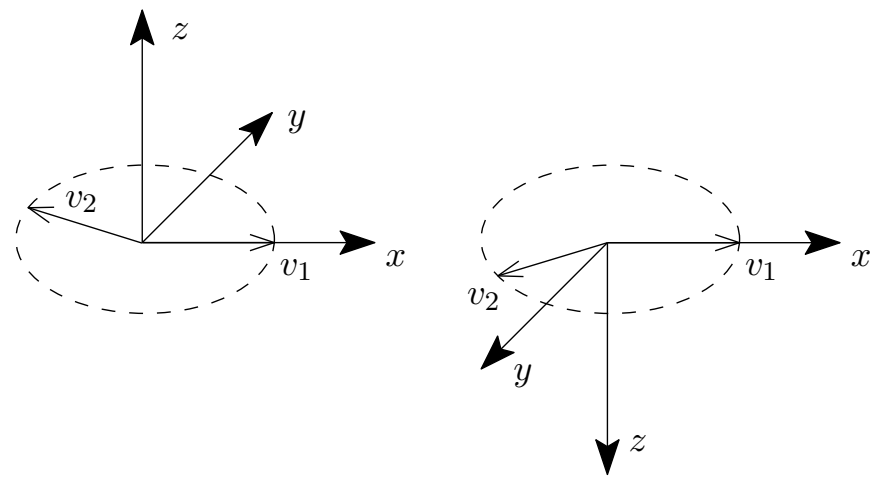

Figure 4. Defined coordinate system using (10)
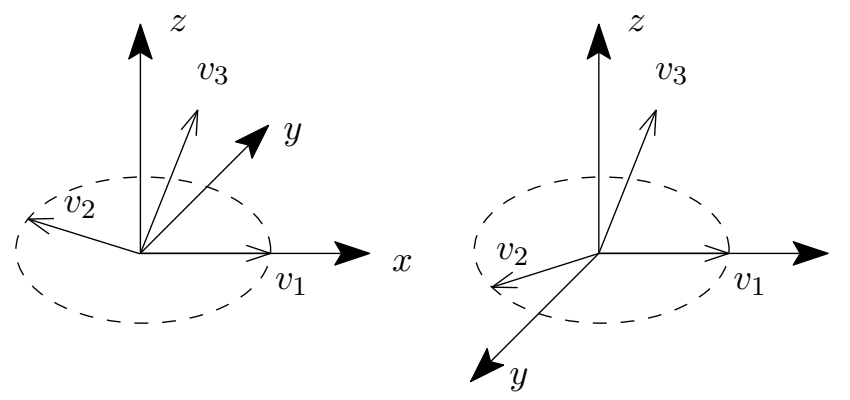

Figure 5. Defined coordinate system using (11)

Let $x^{\mathrm{Gram}}, y^{\mathrm{Gram}}, z^{\mathrm{Gram}}$ be the coordinate axes as in (11). Then define for the coordinate system $T=$ $\left[\begin{array}{lll}x & y & z\end{array}\right]$ as

$$
\begin{aligned}
& x=x^{\mathrm{Gram}}, \\
& y=z \times x, \\
& z=z^{\mathrm{Gram}} .
\end{aligned}
$$

Thus (9) gives with (11) and (12) a method to calculate the continuous angle between $v_{1}$ and $v_{2}$ using an additional vector $v_{3}$. This method is introduced in view of continuous rotations of $v_{1}$ and $v_{2}$ in a slowly changing $v_{1}-v_{2}$-plane. However, it must be ensured that the plane normal does not get perpendicular to $v_{3}$.

\subsubsection{The Solar Noon Angle}

The solar noon angle $\theta$ is the angle between the spacecraft vector $r$ and the sun pointing vector $s$ projected on the orbit plane

$$
\theta=\angle^{x^{\mathrm{SN}}}\left(r,\left(x^{\mathrm{O}} s\right) x^{\mathrm{O}}+\left(z^{\mathrm{O}} s\right) z^{\mathrm{O}}\right),
$$

using (9) and $\left\{x^{\mathrm{SN}}, y^{\mathrm{SN}}, z^{\mathrm{SN}}\right\}$ denoting the coordinate system obtained with Equation (11) and (12) with the vectors $v_{1}=\left(x^{\mathrm{O}} s\right) x^{\mathrm{O}}+\left(z^{\mathrm{O}} s\right) z^{\mathrm{O}}, v_{2}=r$ and $v_{3}=-y^{\mathrm{O}}$. This definition gives for a single orbit of a spacecraft an angle between $(-\pi, \pi]$ with one discontinuity at most.

\subsubsection{The Beta Angle}

The beta angle $\beta \in\left[-\frac{\pi}{2}, \frac{\pi}{2}\right]$ defined as in (Meseguer, Pérez-Grande, and Sanz-Andrés 2012) describes the relative orientation of the orbit with regard to the sun, and is defined as the minimum angle between the orbit plane and the solar vector. The beta angle is defined as positive if the spacecraft orbits in a counter clockwise direction and negative if it revolves clockwise with respect to the sun as

$$
\beta=\left\{\begin{aligned}
\angle\left(s,\left(x^{o} s\right) x^{o}+\left(z^{o} s\right) z^{o}\right) & \text { if } s^{\top} y^{o}<0 \\
-\angle\left(s,\left(x^{o} s\right) x^{o}+\left(z^{o} s\right) z^{o}\right) & \text { if } s^{\top} y^{o} \geq 0
\end{aligned}\right.
$$

using the definition of the orbit frame from Section 2.1 and Equation (8). Another way to calculate the beta angle is to use the normal of the orbit plane and parameterise the vectors by the orbital elements describing the movement of the sun and the satellite. Consider the sun as a satellite of the earth with the 
inclination $i^{s}$ and the sum of the argument of periapsis and true anomaly $\omega^{s}+\nu^{s}$, i.e. the opliquity of the ecliptic and the true solar longitude of the ecliptic. Then the vector to the sun $s$ and the vector orthogonal to the plane $y^{\mathrm{O}}$ can be written in ECI coordinates as:

$$
\begin{aligned}
s= & \cos \left(\omega^{s}+\nu^{s}\right) x^{\mathrm{I}}+\sin \left(\omega^{s}+\nu^{s}\right) \cos \left(i^{s}\right) y^{\mathrm{I}} \\
& +\sin \left(\omega^{s}+\nu^{s}\right) \sin \left(i^{s}\right) z^{\mathrm{I}}, \\
y^{o}= & \sin (\Omega) \sin (i) x^{\mathrm{I}}-\cos (\Omega) \sin (i) y^{\mathrm{I}}+\cos (i) z^{\mathrm{I}} .
\end{aligned}
$$

Instead of calculating the angle to the projection we calculate the angle to the orbit normal as

$$
\begin{aligned}
\sin (\beta)=- & \cos \left(\beta+\frac{\pi}{2}\right)=-s^{\top} y^{o} \\
\Rightarrow \beta=\sin ^{-1}( & \cos \left(\omega^{s}+\nu^{s}\right) \sin (\Omega) \sin (i) \\
& -\sin \left(\omega^{s}+\nu^{s}\right) \cos \left(i^{s}\right) \cos (\Omega) \sin (i) \\
& \left.+\sin \left(\omega^{s}+\nu^{s}\right) \sin \left(i^{s}\right) \cos (i)\right)
\end{aligned}
$$

This description emphasises the dependence of the $\beta$ angle from the orbit inclination and longitude of the ascending node.

\subsubsection{The Solar Zenith Angle}

The solar zenith angle is defined as in (Meseguer, Pérez-Grande, and Sanz-Andrés 2012) to describe the portion of the illuminated planet which is seen by the spacecraft. The solar zenith angle $\xi$ is defined as the angle between the spacecraft vector $r$ and the sun pointing vector $s$ as

$$
\xi=\angle(r, s)
$$

using (8). In order to enable a thermal analysis dependent of the orbit attitude, the influence of this angle can be described by the slowly time varying beta angle $\beta$ and the periodic solar noon angle $\theta$.

For the solar zenith angle $\xi$, the beta angle $\beta$ and the solar noon angle $\theta$ holds

$$
\cos \xi=\cos \beta \cos \theta .
$$

As can be seen in Equation (4) the solar zenith angle influences the acting heat significantly. By using (17) we have introduced two different angles which allow analysing the impact of the chosen satellite orbit. The satellite orbit can be described by the six orbital elements $a, \varepsilon, i, \Omega, \omega$ and $M_{0}$. If the orbiting object is only influenced by a gravitation field described by a spherical symmetric planet these orbital elements are constant. In many applications, orbits are chosen to be circular sun synchronous orbits. Thus, a uniform movement is obtained and the solar noon angle can be described as $\theta=\omega_{o} t$, where $\omega_{o}$ is the angular rotation rate dependent on the semimajor axis $a$. However, the beta angle is determined by the inclination of the orbit $i$ and the of the longitude of the ascending node $\Omega$ as can be seen in (15). Therefore, the choice of $\Omega$ influences the heat acting on the satellite due to the sun significantly.

\subsubsection{The Normal Solar Angle}

The normal solar angle $\phi$ is defined between the normal of a spacecraft surface $n$ and the vector pointing to the sun $s-r$ as

$$
\phi=\angle(s-r, n) \approx \angle(s, n) .
$$

This approximation holds because the distance between earth origin and spacecraft is negligible compared to the distance between sun and spacecraft in low earth orbits.

\subsection{Form Factor}

For the form factor described in the previous section it is sufficient to assume the spacecraft surface to be a infinitesimally small plate and the earth to be a sphere. Then we can use the results from (Juul 1979) and obtain the form factor as a function of distance to the plate and angle $\zeta=\angle(r, n)$, the angle between the normal of the plate $n$ and vector between earth and plate which is approximately the vector between earth and spacecraft $r$. Let $H=\frac{\|r\|}{r_{\oplus}}$ where $r \in \mathbb{R}^{3}$ is the spacecraft position and $r_{\oplus}$ the radius of the earth, then the form factor is

$$
F_{\text {form }}= \begin{cases}\frac{\cos (\zeta)}{H^{2}} & \zeta<\frac{\pi}{2}-\sin ^{-1}\left(\frac{1}{H}\right) \\ F_{\text {form }, 2} & \frac{\pi}{2}-\sin ^{-1}\left(\frac{1}{H}\right)<\zeta<\frac{\pi}{2}+\sin ^{-1}\left(\frac{1}{H}\right) \\ 0 & \zeta>\frac{\pi}{2}+\sin ^{-1}\left(\frac{1}{H}\right)\end{cases}
$$

with

$$
\begin{aligned}
F_{\text {form }, 2}= & \frac{1}{2}-\frac{1}{\pi} \sin ^{-1}\left(\frac{\sqrt{H^{2}-1}}{H \sin (\zeta)}\right) \\
& +\frac{1}{\pi H^{2}}\left(\cos (\zeta) \cos ^{-1}\left(-\sqrt{H^{2}-1} \cot (\zeta)\right)\right. \\
- & \left.\sqrt{H^{2}-1} \sqrt{1-H^{2} \cos (\zeta)^{2}}\right) .
\end{aligned}
$$

\subsection{Shadow Function}

The shadow function gives the occultation of the satellite due to the earth. We use cylindrical shadows as illustrated in Figure 6. The distance between earth and sun is way higher than the difference of their radii and the distance between earth and spacecraft, which is why it is sufficient to assume cylindrical shadows instead of conic ones. We construct an orthonormal basis $\{x, y, z\} \subset \mathbb{R}^{3}$ with $x=\frac{s}{\|s\|}$ then the shadow coefficient $\nu=\nu(r, s)$ is calculated as

$$
\nu= \begin{cases}1 & \text { if } r^{\top} x<0 \wedge\left\|r^{\top} y+r^{\top} z\right\|<r_{\oplus} \\ 0 & \text { otherwise }\end{cases}
$$



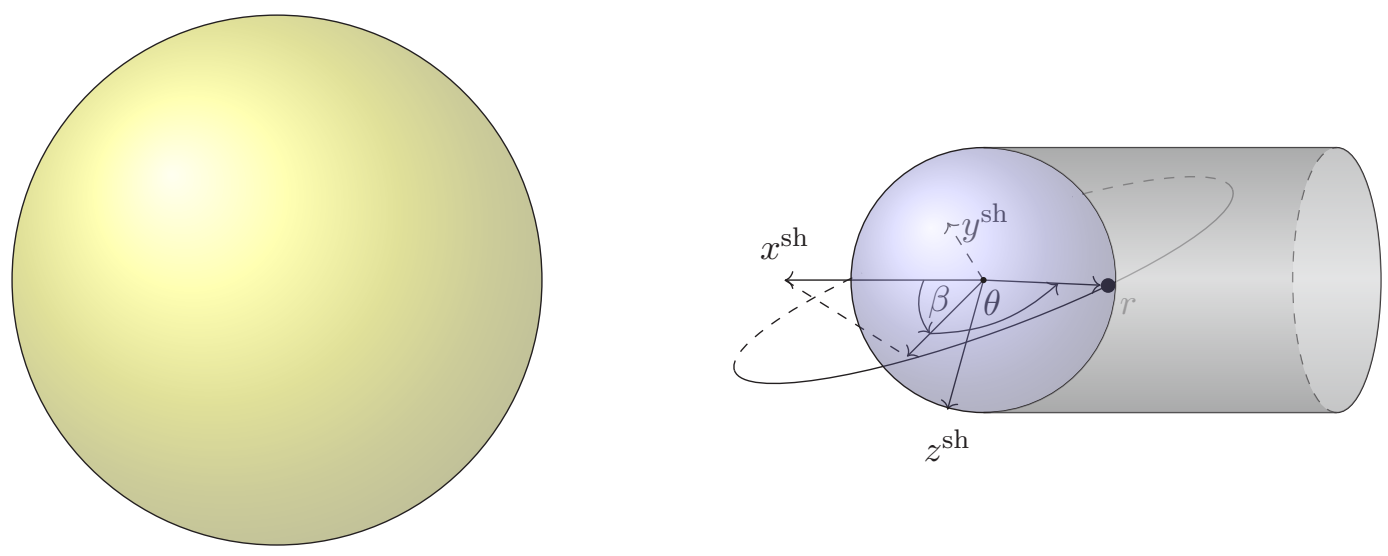

Figure 6. Cylindrical Shadow Model

Note that instead of taking an arbitrary normal basis we can define a coordinate system . $^{\text {sh }}$ using the defined solar noon and beta angle via

$$
\begin{aligned}
T^{\mathrm{sh}, \mathrm{I}} & =\left[\begin{array}{lll}
x^{\mathrm{sh}} & y^{\mathrm{sh}} & z^{\mathrm{sh}}
\end{array}\right]^{\top} \\
& =R_{y}\left(-\frac{\pi}{2}\right) R_{x}(\beta) R_{y}(\theta) R_{y}(\pi) T^{\mathrm{o}, \mathrm{I}},
\end{aligned}
$$

where

$$
R_{x}(\theta)=\left[\begin{array}{ccc}
\cos (\theta) & \sin (\theta) & 0 \\
-\sin (\theta) & \cos (\theta) & 0 \\
0 & 0 & 1
\end{array}\right]
$$

and

$$
R_{y}(\theta)=\left[\begin{array}{ccc}
\cos (\theta) & 0 & \sin (\theta) \\
0 & 1 & 0 \\
-\sin (\theta) & 0 & \cos (\theta)
\end{array}\right] .
$$

We can use this coordinate system to parameterise $r$ as

$\frac{r}{\|r\|}=\cos (\theta) \cos (\beta) x^{\mathrm{sh}}+\cos (\theta) \sin (\beta) y^{\mathrm{sh}}-\sin (\theta) z^{\mathrm{sh}}$.

Then Equation (20) reads

$\nu= \begin{cases}1 & \text { if }|\theta|>\frac{\pi}{2} \wedge \sqrt{\cos (\theta)^{2} \sin (\beta)^{2}+\sin (\theta)^{2}}<\frac{r_{\oplus}}{\|r\|} \\ 0 & \text { otherwise }\end{cases}$

Other methods divide the earth's shadow into umbra and penumbra. The shadow coefficient $\nu \in(0,1)$ in penumbra is then determined by the overlapping of two circular disks. A detailed derivation can be found in (Montenbruck and Gill 2011).

\section{Modelica Implementation}

The implementation of the Thermal Space library is an extension of the DLR Space Systems library from (M. J. Reiner and Bals 2014) and uses gravity and sun models of the DLR Environment Library (Briese, Klöckner, and M. Reiner 2017). The implemented models are based on the Modelica Standard Library.

\subsection{Heat Fluxes Implementation}

Each of the solar radiation, albedo radiation, infrared radiation and deep space radiation is implemented. We will discuss only the implementation of the Albedo radiation in detail as all other radiations follow the same implementation concept. The albedo model is shown in Figure 7 . The user may provide the material specific solar absorptance parameter $\alpha$ as well as the area of the surface $A$ and the normal of the surface $n^{\mathrm{B}}$ in body coordinates. Additionally, the average solar flux constant $G_{s 0}$ and the albedo coefficient $\rho_{\text {alb }}$ may be provided. Standard values for these parameters exist, however it is often desired to simulate special hot and cold case scenarios which makes an adaption of these parameters as implemented a desirable feature. The model has two ports, a frame and a heat port connector. As the spacecraft is usually modelled as a rigid body using the Modelica MultiBody Library (Otter, Elmqvist, and Mattsson 2003), the frame connector has to be connected to the body modelling the spacecraft. Like this the orientation of the frame can be accessed to provide the position $r$ and orientation of the spacecraft $T^{\mathrm{B}}$. Additionally, the outer world model is used to obtain the position of the sun $s$. Then Equations (8) and (16) are used to determine the solar zenith angle $\xi$. The orientation of the spacecraft is used to transform the normal vector in body coordinates $n^{\mathrm{B}}$ into ECI coordinates $n$ using Equation (1). Then the position of the spacecraft $r$ and the normal of the surface $n$ are used to determine the form factor with Equation (19). Finally the albedo heat flow $Q^{\text {alb }}$ is calculated using (4) and fed to the heat port as can be seen in Figure 7. This heat port can then be connected to other sources and sinks of heat to model the thermal dynamics. Instead of using (16), Equation (17) can be used with (9), (12), (13) and (14) to describe the influence of the solar angle. This gives the same results but uses the beta angle $\beta$ instead of the solar zenith angle $\xi$ which may be easier to parameterise with respect to the satellites orbit as can be seen in (15). The other radiations have 

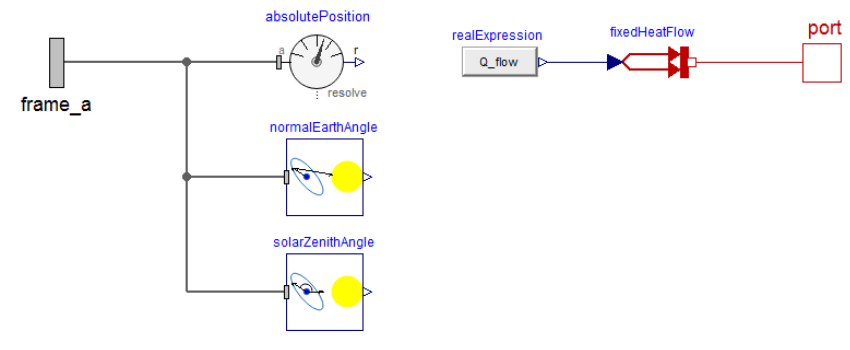

Figure 7. Albedo Model Diagram

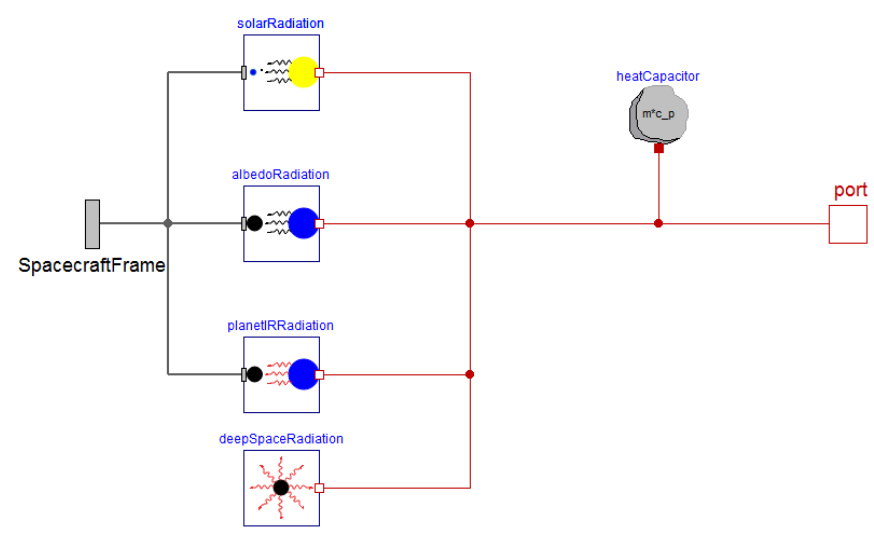

Figure 8. Spacecraft Surface Model Diagram

the same structure but use the Equations (3), (5) and (7), respectively, with the angle defined in (18) and the shadow function (20).

\subsection{Thermal Space Components}

The thermal model of a spacecraft surface can be seen in Figure 8. The thermal dynamics are described by the differential equation

$$
C \dot{T}=Q^{\text {alb }}+Q^{\text {sun }}+Q^{\text {planet }}-\varepsilon A \sigma T^{4}+Q^{r}
$$

where $C$ is the thermal capacitance of the surface and $Q^{r}$ describes all other heat fluxes which are acting on the heat port. This includes foremostly the internal power dissipation of the satellite. The capacitance is implemented as a conditional component. This model offers the opportunity to remove the thermal capacitance if only the steady state calculations are of interest. Additionally, a desired temperature of the surface may be given to obtain the necessary dissipative power which have to be for example produced by heaters to maintain this temperature.

Since many small satellites have the form of a cuboid, a model with six spacecraft surfaces with an infinite resistance between them is implemented. This can be used to simulate the heat evolution at each spacecraft surface as in Section 4. In order to account for the different satellite modes, attitude specific surface configurations are implemented as for e.g. earth pointing mode in which the attitude of the satellite is fixed. Satellite components are modelled as a thermal capacitor which is connected to a spacecraft surface, usually a radiator. For each of these components the parameters already discussed may be provided to simulate different scenarios of interest.

\subsection{Architectures}

There are three thermal concepts commonly used for micro- and nano satellites as described in (Baturkin 2005) - autonomous concept, centralized concept and combined concept. Each of these structures is implemented modelling the thermal coupling between each thermal component and the external heat exchange.

\section{Example Scenario}

In order to show the functionality of the library, the thermal dynamics of a cuboid earth pointing spacecraft are simulated. The cuboid is modelled by six surfaces having the properties of a radiator. The surfaces have the same area $A=1 \mathrm{~m}^{2}$ and thermal properties $\alpha=0.25$ and $\varepsilon=0.88$. The spacecraft is in a sun synchronous orbit with an altitude of $600 \mathrm{~km}$ and $10: 30 \mathrm{~h}$ longitude of the ascending node simulated 2018-02-10 at $10: 00 \mathrm{~h}$. The earth's gravitation field is approximated up to the second zonal coefficient (Markley and Crassidis 2014). No dissipative heat is simulated and the parameter are chosen as $G_{s}=1361 \mathrm{~W} \mathrm{~m}^{-2}, \rho_{\mathrm{alb}}=0.3$ and $T_{p}=255 \mathrm{~K}$. One complete orbit, which takes about $5800 \mathrm{~s} \approx 97 \mathrm{~min}$, is simulated. The satellite is earth pointing over the whole orbit, i.e. the spacecraft body axes which are perpendicular to the cuboid surfaces are aligned with the orbit frame.

Figure 9 shows the visualisation of the described scenario. The spacecraft itself is visualised as a simple grey cuboid. The heat flows, the sum of solar, albedo and infra-red radiation, acting on each surface are visualised using head up displays from the Visualization library (Bellmann 2009). It can be seen that all but the zenith direction are influenced by a constant heat flow due to the earth's infra-red radiation. Furthermore, it can be seen that the spacecraft is in the sunlight after approximately 1100s up to 4990s and that the transition between shadow and sunlit is discontinuous. The nadir direction is mainly influenced by the infra-red and albedo irradiation as its view to sun is mostly blocked by the earth. Due to the low solar absorptance of the surface the change of the acting heat flow is comparatively small. The zenith direction however is mostly influenced by the solar radiation. No albedo and infra-red radiation reaches this surface. The surface perpendicular to the orbit plane and in sun direction is foremostly influenced by the solar radiation as well. However, due to the small change of the angle between this surface and the direction to the sun, this heat flow is almost piecewise constant. On the contrary, the surface perpendicular 


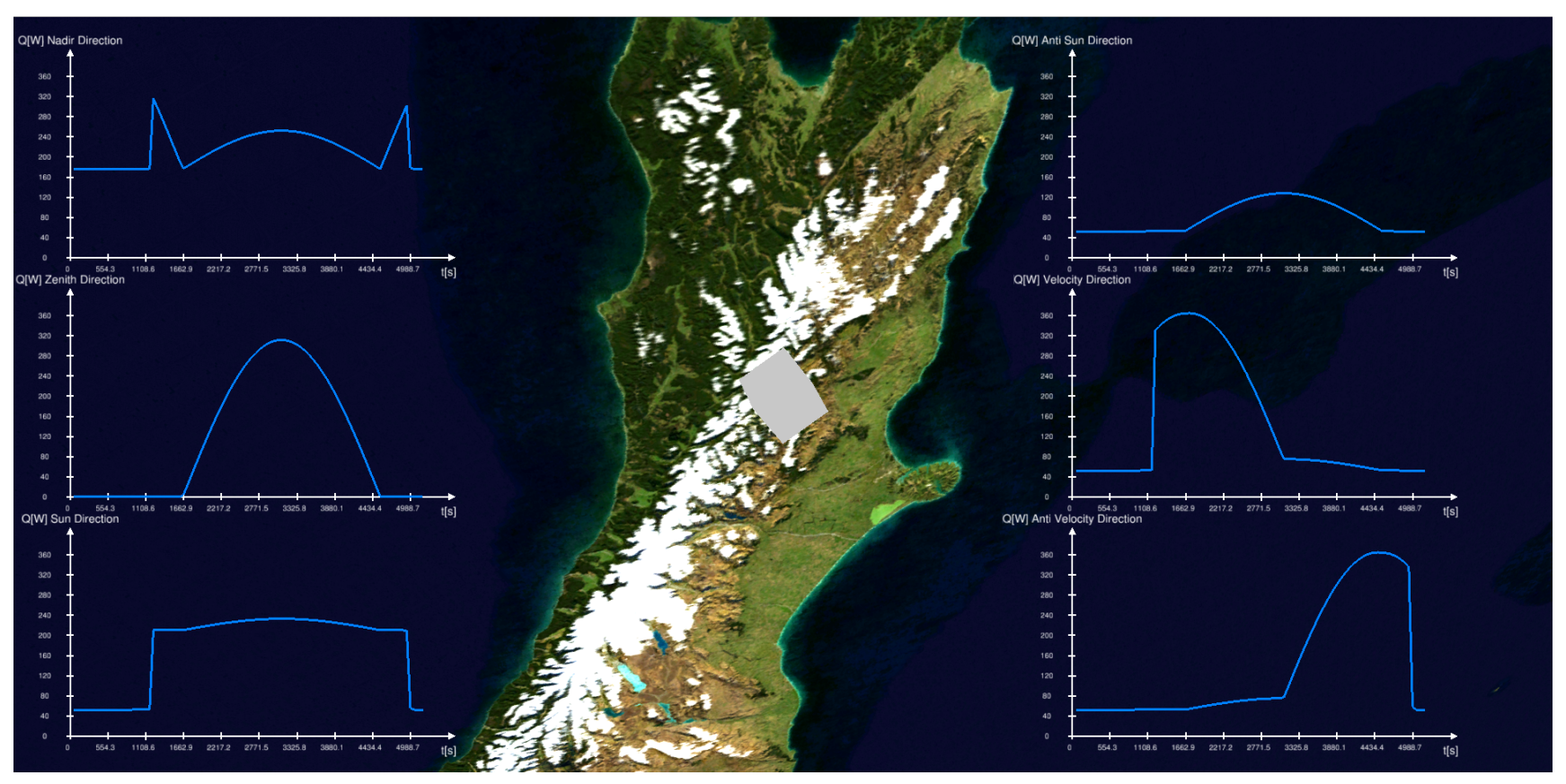

Figure 9. Heat flows acting on the surfaces of a cuboid earth pointing spacecraft

to the orbit plane and in anti sun direction is only influenced by the infra-red and albedo radiation. Due to the small solar absorptance, the albedo radiation influence is comparatively small and this surface has the smallest heat flow changes. The surfaces in velocity and in anti velocity direction are mirrored with respect to the solar noon of the spacecraft. Albedo and infra-red radiation are acting continuously on these surfaces while the influence of the solar radiation can be seen in the sudden discontinuity of the heat flow. All in all, it can be observed that all surfaces are subject to high heat flux changes especially when the spacecraft enters and exits the eclipse. The smallest variation and overall incident heat flux acts on the surface orthogonal to the orbit plane in anti sun direction making it suitable as a surface with radiator.

\section{Conclusions}

We have presented a Modelica library suitable for the development of a thermal spacecraft model. The main acting environmental and spacecraft heat flows are introduced and their dependence on different angles is given as in the literature. Issues regarding the determination of these angles have been described and novel methods for their calculation are given and discussed. An application example of the proposed library is given to demonstrate the usefulness and flexibility of the Modelica implementation.

\section{References}

Baturkin, Volodymyr (2005). "Micro-satellites thermal control-concepts and components". In: Acta Astronautica 56.1-2, pp. 161-170.
Bellmann, Tobias (2009). "Interactive simulations and advanced visualization with modelica". In: Proceedings of the 7th International Modelica Conference; Como; Italy; 20-22 September 2009. 043. Linköping University Electronic Press, pp. 541550.

Briese, Lâle Evrim, Andreas Klöckner, and Matthias Reiner (2017). "The DLR Environment Library for Multi-Disciplinary Aerospace Applications". In: Proceedings of the 12th International Modelica Conference. 132, pp. 929-938.

Fortescue, Peter, Graham Swinerd, and John Stark (2011). Spacecraft systems engineering. John Wiley $\&$ Sons.

Gilmore, David G and Mel Bello (1994). Satellite thermal control handbook. Vol. 1. Aerospace Corporation Press EI Segundo, CA.

Juul, N. H. (1979). "Diffuse Radiation View Factors from Differential Plane Sources to Spheres". In: Journal of Heat Transfer 101.3, p. 558.

Larson, Wiley J. and James R. Wertz (1991). Space Mission Analysis and Design. Springer.

Lefeng, Sun et al. (2017). "Modeling and Simulation on Environmental and Thermal Control System of Manned Spacecraft". In: Proceedings of the 12th International Modelica Conference. 132. Linköping University Electronic Press, pp. 397-405.

Markley, F. Landis and John L. Crassidis (2014). Fundamentals of Spacecraft Attitude Determination and Control. Springer.

Meseguer, J, I Pérez-Grande, and A Sanz-Andrés (2012). Spacecraft Thermal Control. Woodhead Publishing. 
Montenbruck, Oliver and Eberhard Gill (2011). Satellite Orbits: Models, Methods and Applications. Springer.

Otter, Martin, Hilding Elmqvist, and Sven Erik Mattsson (2003). "The New Modelica Multibody Library". In: 3rd International Modelica Conference, pp. 311-330.

Qian, Jing et al. (2015). "Projection-based reducedorder modeling for spacecraft thermal analysis". In: Journal of Spacecraft and Rockets 52.3, pp. 978989.

Reiner, Matthias J and Johann Bals (2014). "Nonlinear inverse models for the control of satellites with flexible structures". In: Proceedings of the 10 th International Modelica Conference. 096, pp. 577-587.

Ruan, Hui, Xiaoguang Hu, and Dan Sun (2017). "Simulation design and implementation of thermal control subsystem for satellite simulator". In: 12th IEEE Conference on Industrial Electronics and Applications. IEEE, pp. 1260-1263.

Tsai, Jih-Run (2004). "Overview of satellite thermal analytical model". In: Journal of spacecraft and rockets 41.1, pp. 120-125. 